\title{
Pramipexole Protects Spinal Motor Neuron Death Against Glutamate-Induced Neurotoxicity
}

\author{
Osamu Kano ${ }^{\mathrm{a}, \mathrm{b}}$, Ken Ikeda ${ }^{\mathrm{a}}$, Kiyokazu Kawabe ${ }^{\mathrm{a}}$, Konosuke Iwamoto ${ }^{\mathrm{a}}$, Yasuo Iwasaki ${ }^{\mathrm{a}}$
}

\begin{abstract}
Background: To determine the possible neuroprotective effect of pramipexole (PPX) against glutamate-induced neurotoxicity, we analyzed the neuroprotective utility of PPX on a postnatal organotypic culture model of rat spinal motor neuron degeneration.

Methods and Results: Treatment with $10^{-5} \mathrm{M}$ of glutamate resulted in a motor neuron loss and decreased activity of choline acetyltransferase (ChAT). Cotreatment of $10^{-5} \mathrm{M}$ of glutamate and PPX revealed a protective effect against motor neuron death and decreased activity of ChAT.

Conclusions: This results show PPX possesses neuroprotective action against glutamate-induced neurotoxicity on spinal motor neuron possibly through a mechanism, which is independent of dopamine receptors but related to antioxidation or scavenging of free radicals. Our data may provide a rationale for designing a therapeutic strategy for neuroprotection against pathologically induced motor neuron death such as amyotrophic lateral sclerosis and spinal motor neuropathy.
\end{abstract}

Keywords: Glutamate-induced neurotoxicity; Amyotrophic lateral sclerosis; Pramipexole; Spinal motor neuron

Manuscript accepted for publication October 17, 2011

${ }^{a}$ Department of Neurology, Toho University Omori Medical Center, 6-11-1 Omorinishi Ota-ku, Tokyo, 143-8541, Japan

bCorresponding author: Osamu Kano, Email: osamukano@aol.com

doi:10.4021/jnr61w

\section{Introduction}

Recent evidence has suggested that on the possible role of oxidative stress in various acute and chronic neurodegenerative disorders [1] Pramipexole (PPX) is a medication that has been used for both Parkinson disease (PD) and restless leg syndrome [2, 3]. Another character of PPX possesses neuroprotective action [4-6].

Amyotrophic lateral sclerosis (ALS) is characterized by loss of motor neurons and etiopathogenesis of this fatal disease is unknown. Glutamate-induced excitotoxicity has been implicated in ALS pathogenesis [1]. Glutamate is the main excitatory neurotransmitter and excessive action of glutamate, known as glutamate-induced excitotoxicity, can induce neurodegeneration through activation of calciumdependent enzyme pathway. Glutamate-induced excitotoxicity can also result in generation of free radicals, which in turn can cause neurodegeneration by damaging intracellular organelles and upregulating proinflammatory mediators [7]. Riluzole is an only drug approved for the drug of ALS, however the extension of survival after treating riluzole is only 3 months [8]. In the other neurodegenerative disorder such as PD, substantial evidence of oxidative stress has also been reported in the causes of PD [9].

Bromocriptine and pergolide, known as dopamine receptor agonists, are widely used for treating PD, and have been reported to be neuroprotective [10,11]. In the present study, using organotypic spinal cord cultures exposed to glutamate, we investigated whether PPX could protect spinal motor neurons against glutamate toxicity.

\section{Methods}

\section{Spinal cord organotypic culture}

Organotypic spinal cord cultures were prepared from lumbar spinal cords of 10-day-old Sprague-Dawely rats (Sankyo Laboratories, Tokyo, Japan). Lumbar spinal cords were removed and sliced into 2-mm-thick transverse sections, and one slice was placed in $3 \mathrm{ml}$ of culture medium in wells 35 

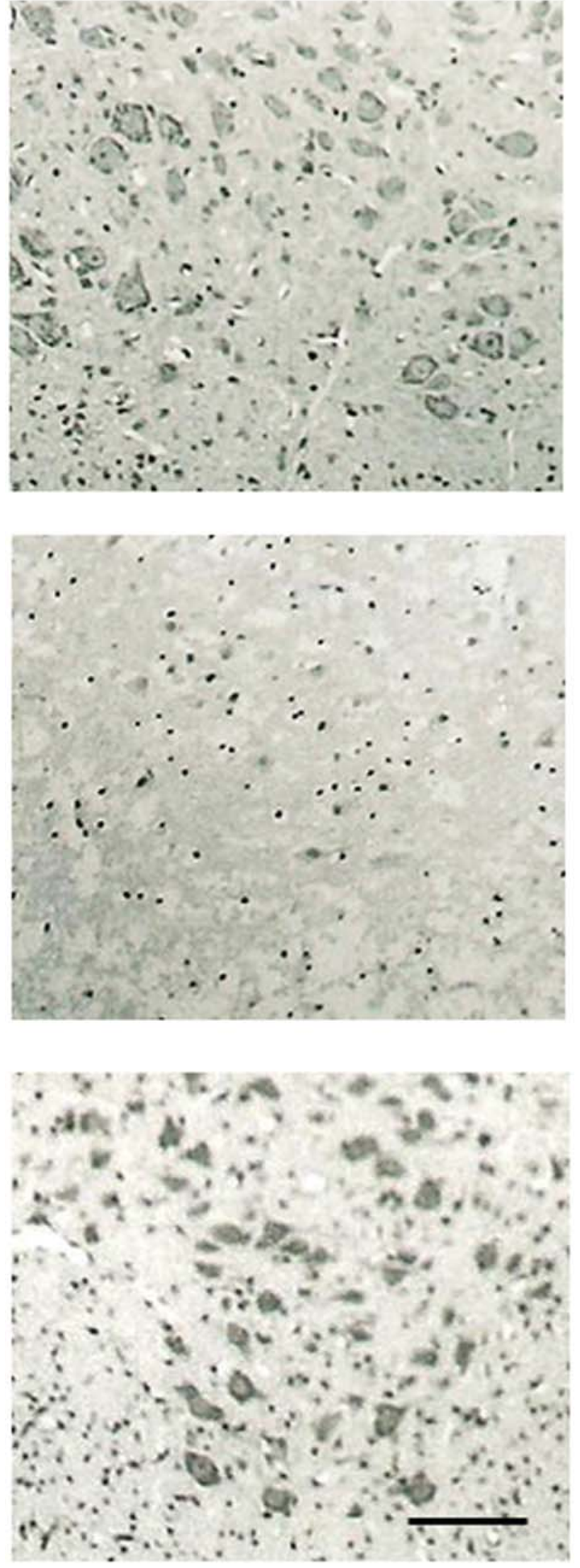

Figure 1. Morphology of spinal motor neurons. Effect of PPX on organotypic cultures of rat spinal cord. Upper: control cultures; Middle: glutamate-treated culture; Lower: co-treatment with glutamate and PPX-treated cultures. (The bar represents $50 \mu \mathrm{m})$; Treatment with glutamtate produced a marked loss of spinal motor neurons compare to control culture; Treatment with PPX protected spinal motor neuron loss against glutamate-induced neurotoxicity. mm in diameter. Culture medium consisted of Delbecco's modified Eagle's medium only. Antibiotics and antifungal agents were not used. Cultures were incubated at $37^{\circ} \mathrm{C}$ and maintained in a humidified atmosphere of 5\% CO2 and 95\% air at $37^{\circ} \mathrm{C}$. The experimental procedure was performed according to the Guidelines of the Animal Committee, Toho University Ohashi Hospital.

\section{Experimental designs}

To determine neuroprotective effects of PPX against glutamate toxicity, cultures were incubated with a dose of $10^{-5}$ M glutamate or glutamate plus PPX for 2 weeks. Culture medium, along with any added pharmacological agents, was exchanged twice weekly. We then compared two groups by counting motor neurons and determining ChAT activity. ChAT staining was performed in the sections of the lumbar segments. The gross morphology of the cultures was monitored daily by inverted Nikon phase-contrast microscopy. To count large motor neurons, cultures were treated with either $10^{-5} \mathrm{M}$ glutamate or glutamate plus $10^{-7}, 10^{-6}$, and $10^{-5} \mathrm{M}$ of PPX for 2 weeks. To determine the number of motor neurons in the spinal cord cultures, we counted neurons meeting following criteria: size ( $>25 \mathrm{um}$ ), possession of at least one thick process, and location in the ventral half of the ventral gray matter [12]. Ten cultures of control and ten from each experimental group were used, and motor neurons were counted randomly by two investigators to exclude the bias of selections.

\section{ChAT activities}

The ChAT activity was also determined 14 days after initial plantings. For the ChAT assay, we used incubation mixture containing $0.2 \mathrm{~mm} 14 \mathrm{C}$ acetyl-CoA (Amersham, Toyama, Japan) $50 \mathrm{mM}$ sodium phosphate buffer (pH 7.4), $300 \mathrm{mM}$ $\mathrm{NaCl}, 8 \mathrm{mM}$ choline chloride, $20 \mathrm{mM}$ EDTA, and $0.1 \mathrm{mM}$ physostigmine. The homogenates ( 2 ul) were put in microtubes, mixed, and incubated for $20 \mathrm{~min}$ at $37^{\circ} \mathrm{C}$. The reaction was stopped by adding $5 \mathrm{ml}$ of $10 \mathrm{mM}$ sodium phosphate buffer (pH 7.4), and the contents were transferred to scintillation vials. Then $2 \mathrm{ml}$ of acetonitrile containing $10 \mathrm{mg}$ of sodium tetraphenylborone and $10 \mathrm{ml}$ of toluene scintillation mixture (0.4\% DPO, 0.01\% POPOP) were added to the vials. All vials were counted using a liquid scintillation counter (Aloka LC-3500). The protein concentration was determined by a Bio-Rad protein assay kit with bovine serum albumin as standard. The enzyme activity was expressed as acetylcholine production per milligrams of protein per minute.

\section{Statistical analyses}

Results were expressed by mean \pm SEM of a representive experiment. Statistical analyses were done with Mann-Whit- 


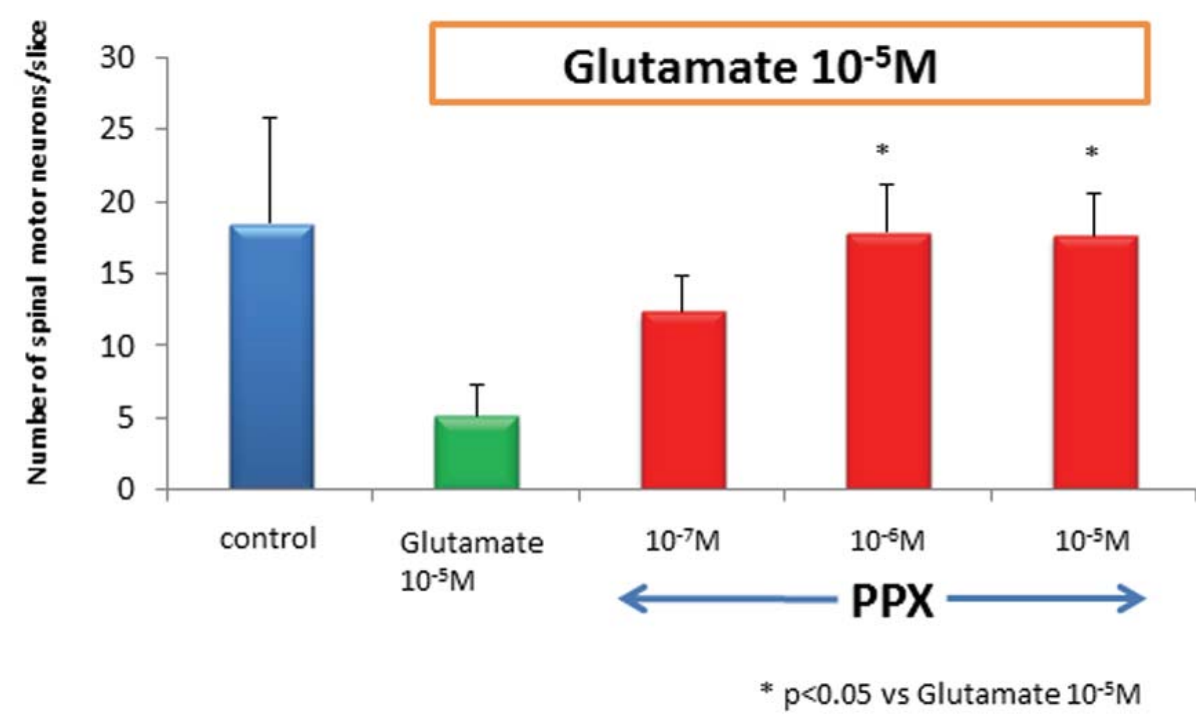

Figure 2. Number of spinal motor neurons. After 2 weeks of cultures, the number of spinal motor neurons in glutamate-treated cultures dropped by approximately $80 \%$ as compared to control. PPX act $10^{-6} \mathrm{M}$ and $10^{-5} \mathrm{M}$ protected spinal motor neurons against glutamate-induced neurotoxicity.

ney's U-test. The significance level was set at 0.05 for all tests. Ten cultures of control and six from each experimental group were analyzed with ChAT activities.

\section{Results}

We counted motor neurons in ChAT staining that had been treated with control medium, glutamate alone, or PPX plus glutamate. The variance among treatment group was highly significant, in which glutamate killed a median $80 \%$ of spinal motor neurons when compared to untreated control. PPX treatment of glutamate-induced neurotoxicity cultures virtually completely spared spinal motor neurons (Fig. 1). There was no significant difference for the surviving spinal motor neurons between $10^{-5} \mathrm{M}$ of glutamate and glutamate plus $10^{-7} \mathrm{M}$ of PPX. This means $10^{-7} \mathrm{M}$ of PPX could not prevent the loss of motor neurons. The addition of $10^{-6}$ and $10^{-5} \mathrm{M}$ of

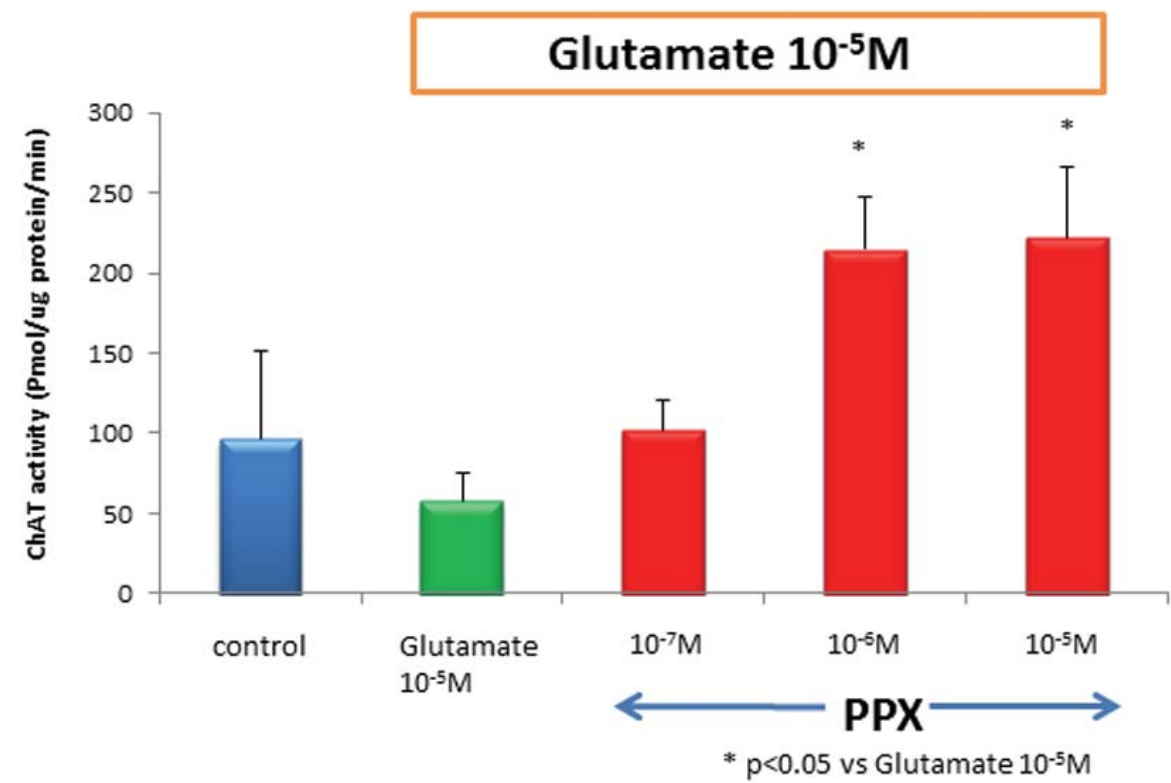

Figure 3. ChAT activity treatment of cultures with $10^{-5} \mathrm{M}$ glutamate reduced ChAT activity approximately to $40 \%$ of untreated control. However, cotreatment of $10^{-6} \mathrm{M}$ of PPX and glutamate was significantly neuroprotective, compared with glutamate-induced neurotoxicity. Culture treatment was 2 weeks. 
PPX in glutamate-treated cultures showed significant neuroprotective effect (Fig. 2). ChAT activity was determined as a surrogate biochemical marker of spinal motor neuron survival in toxicity and neuroprotective experiments. ChAT activity in glutamate-treated cultures dropped approximately $50 \%$ when compared to that of untreated spinal cord cultures. In cultures treating with $10^{-6} \mathrm{M}$ of PPX and glutamate, the decrease in ChAT activity was prevented (Fig. 3).

\section{Discussion}

In this model, we used two independent methods which show the neuroprotective action of PPX on glutamate induced neurotoxicity in the spinal cord cultures. First we counted the number of motor neurons in organotypic cultures. Second, we used a biochemical assay to measure ChAT activity, because ChAT is a reliable marker of large spinal motor neurons.

Neuroprotective effect of PPX on glutamate-induced neurotoxicity was not identical between number of spinal motor neurons and ChAT activity, and ChAT activity was increased more in cultures treated with glutamate plus PPX than those in control cultures, indicating that PPX treatment enhances biochemical effects rather than morphological counter parts.

Our results showed that PPX possess significant neuroprotective effect in spinal motor neuron against glutamateinduced neurotoxicity. Glutamate induced neurotoxicity could be mediated by both NMDA and non-NMDA glutamate receptor subtypes. Spinal motor neurons possess both NMDA and non-NMDA receptors. Glutamate induces various neurotoxic cellular processes, such as formation of oxygen radicals or calcium release [7]. Glutamate metabolism plays an important role in the pathogenesis of motor neuron degeneration, such as ALS $[1,13]$.

Our results showed that PPX might play a neuroprotective role in glutamate induced neurotoxicity. However, the exact mechanism of neuroprotection in PPX against glutamate-induced neurotoxicity is elusive.

Several studies have reported antioxidation or scavenging of free radicals for dopamine agonists, including apomorphine, bromocriptine, pergolide, PPX and ropinirole in cell cultures and animal model of injury of substantia nigra [14-23]. Preincubation of primary mesencephalic neuronal cultures with bromocriptine or quinpirole has provided neuroprotection against glutamate-induced neurotoxicity [24]. These findings indicate that dopamine agonists provide synthesis of proteins that scavenge free radicals via dopamine receptor stimulation.

In contrast, other group has found dopamine receptors are not required to mediate the neuroprotective action of dopamine agonists [25]. For example, the inactive stereoisomers of PPX and apomorphine still have shown neuro- protective action in in vitro models despite their inability to activate dopamine receptors [26, 27]. Dopamine receptor blockers, such as sulpiride and clozapine, do not prevent the neuroprotective action of PPX. Moreover, dopamine agonist has protective activity in cells devoid of dopamine receptors. Izumi et al. reported that PPX protects dopaminergic neurons from glutamate neurotoxicity by the reduction of intracellular dopamine content, independently of dopamine $\mathrm{D}_{2}$-like receptor activation [28].

In spinal cord, we have reported both pergolide and bromocriptine possess neuroprotective action on spinal motor neurons against axotomy-induced spinal motor neuron death and neuron damage following inhibition of superoxide dismutase $[10,11]$.

Glutamate transporters play an important role in keeping extracellular glutamate concentrations below neurotoxic levels and their blockade causes neuronal death [29]. Yamashita et al. studied the effect of bromocriptine on glutamate transport via the human glutamate transporter, hGluT-1, and demonstrated that bromocriptine enhances glutamate uptake. Therefore, they suggested that bromocriptine modulated glutamate transported through an allosteric effect and enhanced the affinity of hGluT-1 for glutamate. In addition, the cells were not provided with dopamine receptors, which meant the effect was not modulated by dopamine receptors [30].

This is the first report that PPX possess significant neuroprotective effect in spinal motor neuron against glutamateinduced neurotoxicity. These evidences and our results suggest that PPX may have neuroprotective effect on spinal motor neuron possibly through a mechanism, which is independent of dopamine receptors but related to antioxidation or scavenging of free radicals. Further studies are needed to elucidate the role of dopamine agonists against motor neurons.

\section{Acknowledgement}

This work was supported by grants from The Uehara Memorial Foundation and Kanae Foundation for the promotion of medical science.

\section{Conflict of Interest}

All authors report no conflicts of interest.

\section{References}

1. Iwasaki Y, Ikeda K, Kinoshita M. Molecular and cellular mechanism of glutamate receptors in relation to amyotrophic lateral sclerosis. Curr Drug Targets CNS Neurol Disord. 2002;1(5):511-518. 
2. Kano O, Ikeda K, Kiyozuka T, Iwamoto K, Ito H, Kawase Y, Sato R, et al. Beneficial effect of pramipexole for motor function and depression in Parkinson's disease. Neuropsychiatr Dis Treat. 2008;4(4):707-710.

3. Aiken CB. Pramipexole in psychiatry: a systematic review of the literature. J Clin Psychiatry. 2007;68(8):1230-1236.

4. Albrecht S, Buerger E. Potential neuroprotection mechanisms in PD: focus on dopamine agonist pramipexole. Curr Med Res Opin. 2009;25(12):2977-2987.

5. Lemke MR, Brecht HM, Koester J, Reichmann H. Effects of the dopamine agonist pramipexole on depression, anhedonia and motor functioning in Parkinson's disease. J Neurol Sci. 2006;248(1-2):266-270.

6. Gribkoff VK, Bozik ME. KNS-760704 (6R)-4,5,6,7-tetrahydro-N6-propyl-2, 6-benzothiazole-diamine dihydrochloride monohydrate for the treatment of amyotrophic lateral sclerosis. CNS Neurosci Ther. 2008;14(3):215226.

7. Culcasi M, Lafon-Cazal M, Pietri S, Bockaert J. Glutamate receptors induce a burst of superoxide via activation of nitric oxide synthase in arginine-depleted neurons. J Biol Chem. 1994;269(17):12589-12593.

8. Bensimon G, Lacomblez L, Meininger V. A controlled trial of riluzole in amyotrophic lateral sclerosis. ALS/ Riluzole Study Group. N Engl J Med. 1994;330(9):585591.

9. Jenner P. Oxidative stress as a cause of Parkinson's disease. Acta Neurol Scand Suppl. 1991;136:6-15.

10. Iwasaki Y, Ikeda K, Shiojima T, Kobayashi T, Tagaya N, Kinoshita M. Deprenyl and pergolide rescue spinal motor neurons from axotomy-induced neuronal death in the neonatal rat. Neurol Res. 1996;18(2):168-170.

11. Iwasaki Y, Ikeda K, Shiojima T, Tagaya N, Kobayashi T, Kinoshita M. Bromocriptine prevents neuron damage following inhibition of superoxide dismutase in cultured ventral spinal cord neurons. Neurol Res. 1997;19(4):389392.

12. Bilak MM, Corse AM, Bilak SR, Lehar M, TombranTink J, Kuncl RW. Pigment epithelium-derived factor (PEDF) protects motor neurons from chronic glutamatemediated neurodegeneration. J Neuropathol Exp Neurol. 1999;58(7):719-728.

13. Leigh PN, Meldrum BS. Excitotoxicity in ALS. Neurology. 1996;47(6 Suppl 4):S221-227.

14. Finotti N, Castagna L, Moretti A, Marzatico F. Reduction of lipid peroxidation in different rat brain areas after cabergoline treatment. Pharmacol Res. 2000;42(4):287291.

15. Gassen M, Glinka Y, Pinchasi B, Youdim MB. Apomorphine is a highly potent free radical scavenger in rat brain mitochondrial fraction. Eur J Pharmacol. 1996;308(2):219-225.

16. Gomez-Vargas M, Nishibayashi-Asanuma S, Asanuma M, Kondo Y, Iwata E, Ogawa N. Pergolide scavenges both hydroxyl and nitric oxide free radicals in vitro and inhibits lipid peroxidation in different regions of the rat brain. Brain Res. 1998;790(1-2):202-208.

17. Jenner P. Pharmacology of dopamine agonists in the treatment of Parkinson's disease. Neurology. 2002;58(4 Suppl 1):S1-8.

18. Muralikrishnan D, Mohanakumar KP. Neuroprotection by bromocriptine against 1-methyl-4-phenyl-1,2,3,6-tetrahydropyridine-induced neurotoxicity in mice. FASEB J. 1998;12(10):905-912.

19. Nishibayashi S, Asanuma M, Kohno M, Gomez-Vargas M, Ogawa N. Scavenging effects of dopamine agonists on nitric oxide radicals. J Neurochem. 1996;67(5):22082211.

20. Ogawa N, Tanaka K, Asanuma M, Kawai M, Masumizu T, Kohno M, Mori A. Bromocriptine protects mice against 6-hydroxydopamine and scavenges hydroxyl free radicals in vitro. Brain Res. 1994;657(1-2):207-213.

21. Yoshioka M, Tanaka K, Miyazaki I, Fujita N, Higashi Y, Asanuma M, Ogawa N. The dopamine agonist cabergoline provides neuroprotection by activation of the glutathione system and scavenging free radicals. Neurosci Res. 2002;43(3):259-267.

22. Clow A, Freestone C, Lewis E, Dexter D, Sandler M, Glover V. The effect of pergolide and MDL 72974 on rat brain CuZn superoxide dismutase. Neurosci Lett. 1993;164(1-2):41-43.

23. Le WD, Jankovic J. Are dopamine receptor agonists neuroprotective in Parkinson's disease? Drugs Aging. 2001;18(6):389-396.

24. Sawada H, Ibi M, Kihara T, Urushitani M, Akaike A, Kimura J, Shimohama S. Dopamine D2-type agonists protect mesencephalic neurons from glutamate neurotoxicity: mechanisms of neuroprotective treatment against oxidative stress. Ann Neurol. 1998;44(1):110119.

25. Zou L, Jankovic J, Rowe DB, Xie W, Appel SH, Le W. Neuroprotection by pramipexole against dopamine- and levodopa-induced cytotoxicity. Life Sci. 1999;64(15):1275-1285.

26. Gassen M, Gross A, Youdim MB. Apomorphine enantiomers protect cultured pheochromocytoma (PC12) cells from oxidative stress induced by H2O2 and 6-hydroxydopamine. Mov Disord. 1998;13(2):242-248.

27. Schapira AH. Neuroprotection in PD--a role for dopamine agonists? Neurology. 2003;61(6 Suppl 3):S34-42.

28. Izumi Y, Sawada H, Yamamoto N, Kume T, Katsuki H, Shimohama S, Akaike A. Novel neuroprotective mechanisms of pramipexole, an anti-Parkinson drug, against endogenous dopamine-mediated excitotoxicity. Eur J Pharmacol. 2007;557(2-3):132-140.

29. Kawakami H, Tanaka K, Nakayama T, Inoue K, Nakamura S. Cloning and expression of a human glutamate transporter. Biochem Biophys Res Commun. 
1994;199(1):171-176.

30. Yamashita H, Kawakami H, Zhang YX, Tanaka K, Na- kamura S. Neuroprotective mechanism of bromocriptine. Lancet. 1995;346(8985):1305. 